Cahiers québécois de démographie

\title{
La politique de répartition géographique des effectifs médicaux au Québec
}

The Policy of Geographical Distribution of Quebec's Medical Staff

\section{La politica de distribucion geografica del personal médico, en Québec}

\section{Richard Boulard et Desmond Dufour}

Volume 12, numéro 1, avril 1983

La mortalité

URI : https://id.erudit.org/iderudit/600491ar

DOI : https://doi.org/10.7202/600491ar

Aller au sommaire du numéro

Éditeur(s)

Association des démographes du Québec

ISSN

0380-1721 (imprimé)

1705-1495 (numérique)

Découvrir la revue

Citer cet article

Boulard, R. \& Dufour, D. (1983). La politique de répartition géographique des effectifs médicaux au Québec. Cahiers québécois de démographie, 12(1), 83-105. https://doi.org/10.7202/600491ar
Résumé de l'article

Un des objectifs du Ministère des Affaires sociales est d'assurer le plus possible à tous un égal accès aux soins de santé. Une évaluation quantitative de la répartition des médecins montre qu'il existe des disparités régionales importantes. Les médecins sont fortement concentrés dans les régions universitaires de Montréal, Québec et Sherbrooke, ce qui n'assure pas à la population de certaines régions périphériques l'accessibilité visée. Plusieurs solutions ont été envisagées à la suite de l'analyse des causes de ce déséquilibre régional des effectifs médicaux. Finalement, pour corriger cette situation, le Gouvernement a élaboré et mis en place une politique de répartition axée principalement sur une rémunération différente des médecins selon les lieux d'exercice. Quelques résultats préliminaires de cette politique sont présentés dans la dernière partie. 
Cahiers québécois de démographie

Vol. 12, no 1, avril 1983

LA POLITIQUE DE REPARTITION GEOGRAPHIQUE

DES EFFECTIFS MEDICAUX AU QUEBEC

Richard BOULARD et Desmond DUFOUR*

Introduction

Depuis plusieurs années, des citoyens de certaines régions éloignées se sont plaints des services médicaux qui leur étaient offerts. Ces plaintes ne mettaient pas en cause la compétence des médecins, mais les difficultés à recevoir les soins de santé qu'ils réclamaient en raison du nombre insuffisant de médecins.

Dans ces mêmes régions, les médecins se sont plaints de leur charge de travail beaucoup plus lourde et exigeante que celle d'un médecin de Montréal. Cela a provoqué, à l'occasion, des crises particulièrement pénibles, par exemple lors de la démission des médecins d'un hôpital en vue de sensibiliser leurs confrères à la situation de pénurie. Face à celle ci, une politique a été mise en place par le ministère des Affaires sociales dans le but de modifier la répartition géographique des médecins. Cette mesure ne vise ni la croissance ni la décroissance de la population médicale, mais une certaine équité dans sa distribution.

La politique proposée découle du principe de base de notre système de santé qui vise à assurer le plus possible à toute la population un égal accès aux soins. Au cours des dernières années, la répartition des effectifs médicaux $n^{\prime} a$ pas permis de respecter ce principe. Les médecins ont tendance à s'installer dans les grands centres urbains tarditionnellement bien pourvus en professionnels de la santé au détriment des régions périphériques et éloignées.

L'objectif de cette politique en ce qui a trait aux omnipraticiens vise à atteindre, dans toutes les régions, un nombre de médecins généralistes suffisant pour assurer les services médicaux de base et à constituer des équipes permanentes intégrées dans leur milieu. Pour ce qui est des médecins spécialistes, l'objectif est de constituer des équipes stables et permanentes avec un éventail plus grand de spécialités: cette politique devrait accroitre le nombre de spécialistes, diminuer la mobilité du personnel médical et réduire les services épisodiques (1) rendus par des médecins itinérants.

* Ministère des Affaires sociales.

(1) Services médicaux rendus dans les régions en pénurie par les spécialistes des régions en surplus. 
En première partie, nous analysons l'évolution de la répartition régionale des médecins omnipraticiens et spécialistes entre 1972 et 1980. Les causes de la mauvaise répartition géographique des médecins sont exposées dans la deuxième partie, alors que les hypothèses de solution font l'objet du troisième point. Les principes et le contenu de la politique de répartition sont présentés dans la quatrième partie. Enfin, des résultats préliminaires complètent cette analyse.

\section{La répartition géographique des médecins} 1.1 Evolution de _la répartition régionale des médecins de 1972 à

Au Québec, en 1972, il y avait 7049 médecins tandis qu'en 1980, on en comptait 11 052, soit une augmentation de 57\%. Durant cette même période, la population québécoise augmentait de 5,1\% seulement. L'augmentation du nombre de médecins a donc été en huit ans onze fois supérieure à celle de la population totale. Cette croissance rapide des effectifs médicaux aurait da avoir pour effet de doter le Québec d'un nombre suffisant de médecins pour répondre aux besoins de la population dans toutes les régions du Québec.

La norme proposée par le Comité national de la main d'oeuvre médicale (1975) (2) pour 1981 est de 1 médecin omnipraticien pour 1440 habitants. Non seulement, selon cette norme, le Québec avait-il en 1980 suffisamment de médecins, soit 5505 omnipraticiens, incluant les résidents (tableau 1) et un ratio de 1 pour 1156 habitants, mais on pourrait même parler d'un surplus d'environ 1000 médecins omnipraticiens, soit 19\% de l'effectif total (tableau 2).

Une comparaison du ratio population/spécialiste avec les normes du Comité national de la main d'oeuvre médicale montre que, parmi les 23 spécialités étudiées, quinze présentent des surplus et huit, des pénuries (tableau 2). Globalement, le Québec avait suffisamment de médecins spécialistes en 1980; il dépasse même la norme fédérale d'environ 500 spécialistes, soit $9 \%$ de l'effectif total.

Bien que le nombre total de médecins soit suffisant, nous observons depuis quelques années une concentration de plus en plus grande des effectifs médicaux dans les grands centres urbains, tandis que plusieurs régions éloignées souffrent d'une pénurie chronique de médecins.

(2) Cette norme, établie pour l'année 1981, tient compte de toutes les activités professionnelles des médecins (pratique médicale, enseignement, recherche, administration....). 
TABLEAU 1: NOMBRE DE MEDEC INS OMNIPRATICIENS ET SPECIALISTES ET RATIO POPULATION/MEDECIN, REGIONS SOCIO-SANITAIRES, QUEBEC 1972 ET 1980

OMNIPRATICIENS ${ }^{(1)}$

\begin{tabular}{|c|c|c|c|c|c|c|c|}
\hline \multicolumn{2}{|r|}{ REGIONS } & \multicolumn{2}{|c|}{ NOMBRE 1972 RATIO } & \multicolumn{2}{|c|}{${ }_{\text {NOMBRE }}^{1380}$ RATIO } & \multicolumn{2}{|c|}{$\begin{array}{lc}\text { ACCROISSEMENT } & 1972-1980 \\
\text { NOMBRE } & \%\end{array}$} \\
\hline $\begin{array}{c}01 \\
02 \\
03 \\
04 \\
05 \\
06 \\
06 \mathrm{~A} \\
06 \mathrm{~B} \\
06 \mathrm{C} \\
07 \\
08 \\
09+10\end{array}$ & $\begin{array}{l}\text { Bas-St-Laurent-Gaspésie } \\
\text { Saguenay-Lac-St-Jean } \\
\text { Québec } \\
\text { Trois-Rivières } \\
\text { Cantons-de-l'Est } \\
\text { Montréal (ensemble) } \\
\text { Mont.-métro. et Laval } \\
\text { Laurentides-Lanaudière } \\
\text { Montérégie } \\
\text { Outaoua is } \\
\text { Nord-Ouest } \\
\text { Côte-Nord et N.-Ouébec }\end{array}$ & $\begin{array}{rr} & 94 \\
& 97 \\
& 497 \\
170 \\
\\
100 \\
1 \quad 847 \\
1 \quad 247 \\
& 175 \\
& 425 \\
& 86 \\
& 46 \\
& 42\end{array}$ & $\begin{array}{ll}2 & 447 \\
2 & 874 \\
1 & 908 \\
2 & 459 \\
2 & 242 \\
1 & 870 \\
1 & 757 \\
2 & 297 \\
2 & 025 \\
2 & 870 \\
3 & 136 \\
2 & 742\end{array}$ & 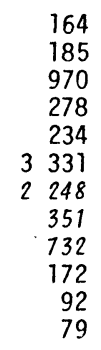 & $\begin{array}{ll}1 & 374 \\
1 & 555 \\
1 & 043 \\
1 & 535 \\
& 999 \\
1 & 080 \\
& 911 \\
1 & 423 \\
1 & 434 \\
1 & 679 \\
1 & 661 \\
1 & 751\end{array}$ & $\begin{array}{rr} & 70 \\
& 88 \\
& 473 \\
& 108 \\
& 134 \\
1 & 484 \\
1 & 001 \\
& 176 \\
& 307 \\
& 86 \\
& 46 \\
& 37\end{array}$ & $\begin{array}{r}74,5 \\
90,7 \\
95,2 \\
63,5 \\
134,0 \\
80,0 \\
80,3 \\
100,6 \\
72,2 \\
100,0 \\
100,0 \\
88,1\end{array}$ \\
\hline & TOTAL & 2979 & 2033 & 5505 & 1156 & 2526 & 84,8 \\
\hline & ECARTS & $08 / 06 \mathrm{~A}$ & $=1,8$ & $08 / 06 \mathrm{~A}$ & $=1,8$ & & \\
\hline
\end{tabular}

SPECIALISTES ${ }^{(2)}$

\begin{tabular}{|c|c|c|c|c|c|c|c|}
\hline \multicolumn{2}{|r|}{ REGIONS } & \multicolumn{2}{|c|}{${ }_{\text {NOMBRE }}^{1972}$ RAT IO } & \multicolumn{2}{|c|}{${ }_{\text {NOMBRE }}^{1980}$ RATIO } & \multicolumn{2}{|c|}{$\begin{array}{cc}\text { ACCRO ISSEMENT } & 1972-1980 \\
\text { NOMBRE } & \%\end{array}$} \\
\hline $\begin{array}{l}01 \\
02 \\
03 \\
04 \\
05 \\
06 \\
06 \mathrm{~A} \\
06 \mathrm{~B} \\
06 \mathrm{C} \\
07 \\
08 \\
09+10\end{array}$ & $\begin{array}{l}\text { Bas-St-Laurent-Gaspésie } \\
\text { Saguenay-Lac-St-Jean } \\
\text { Québec } \\
\text { Trois-Rivières } \\
\text { Cantons-de-l'Est } \\
\text { Montréal (ensemble) } \\
\text { Mont.-métro. et Laval } \\
\text { Laurentides-Lanaudiēre } \\
\text { Montérégie } \\
\text { Outaouais } \\
\text { Nord-Ouest } \\
\text { Côte-Nord et N. -Ouébec }\end{array}$ & $\begin{array}{r}61 \\
111 \\
627 \\
178 \\
193 \\
2806 \\
- \\
- \\
- \\
52 \\
29 \\
13\end{array}$ & 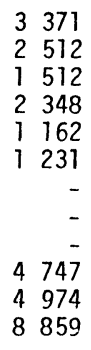 & 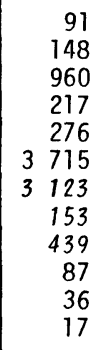 & $\begin{array}{ll}2 & 476 \\
1 & 944 \\
1 & 053 \\
1 & 966 \\
& 847 \\
& 968 \\
& 656 \\
3 & 266 \\
2 & 391 \\
3 & 320 \\
4 & 244 \\
8 & 139\end{array}$ & $\begin{array}{r}30 \\
37 \\
333 \\
39 \\
83 \\
909 \\
- \\
- \\
- \\
35 \\
7 \\
4\end{array}$ & $\begin{array}{r}49,2 \\
33,3 \\
53,1 \\
21,9 \\
43,0 \\
32,4 \\
- \\
- \\
- \\
67,3 \\
24,7 \\
30,8\end{array}$ \\
\hline & TOTAL & 4070 & 1488 & 5547 & 1147 & 1477 & 36,3 \\
\hline & ECARTS & $08 / 0$ & $=4,3$ & $08 / 0$ & $=5,0$ & & \\
\hline
\end{tabular}

SOURCES: REGIE DE L'ASSURANCE-MALADIE DU OUEBEC, Troisième rapport 1971-1972, tableau K. REGIE DE L'ASSURAIICE-MALADIE DU OUEBEC, Statistiques annuelles 1980, pp. 90-91. MINISTERE DES AFFAIRES SOCIALES, Super-Pop, mars 1976; Fécond ité faible et migration = hypothèse $B$ (pour les données de 1972).

MINISTERE DES AFFAIRES SOCIALES, Super-Pop, résultats révisés du modẽle Super-Pop, juillet 1980; Fécondité faible et migration = hypothèse B (pour les données de 1980).

(1) Les données comprennent tous les omnipraticiens et les résidents rémunérês par 1a R.A.M.O.

(2) Ensemble des 31 spécialités rémunêrêes par la R.A.M.0. 
TABLEAU 2: NOHBRE DE MEDECINS ET RATIO POPULATIOM/MEDECIN EN 1980,

NORMES FEDERALES POUR 1981 ET PENURIES-SURPLUS SELON LES NORMES, QUEBEC 1980

\begin{tabular}{|c|c|c|c|c|c|}
\hline SPECIALITE & $\begin{array}{l}\text { Nombrè de } \\
\text { médecins } \\
1980\end{array}$ & $\begin{array}{l}\text { Ratio } \\
\text { pop./medec in } \\
1980\end{array}$ & $\begin{array}{l}\text { Norme féderale } \\
\text { pour } 1981\end{array}$ & $\begin{array}{l}\text { Nombre de medecins } \\
\text { renuis selon la } \\
\text { norme féderale }\end{array}$ & $\begin{array}{l}\text { Penurie }(-) \\
\text { Surplus }(+)\end{array}$ \\
\hline Ana tomo-pathologie & 195 & 32638 & 16100 & 395 & -200 \\
\hline Anesthessie-réanimation & 430 & 14801 & 13742 & 463 & -33 \\
\hline Biochinie médicale & 29 & 219465 & 204000 & 31 & -2 \\
\hline Chirurgie cardio-vasculaire & 57 & 111658 & 117000 & 54 & +3 \\
\hline Chirurgie génerale & 599 & 10625 & 11000 & 579 & +20 \\
\hline Chirurgie orthopedique & 244 & 26084 & 30400 & 209 & +35 \\
\hline Chirurgie plastique & 81 & 78574 & 100000 & 64 & +17 \\
\hline Dermatologie & 103 & 61791 & 65300 & 97 & +6 \\
\hline Medecine interne regroupee (1) & 1148 & 5544 & 8200 & 776 & +372 \\
\hline Médecine nucleaire & 31 & 205306 & 250000 & 25 & +6 \\
\hline Microbiologie médicale & 77 & 82656 & 100000 & 64 & +13 \\
\hline Neurochirurgie & 54 & 117861 & 166500 & 38 & +16 \\
\hline Neurologie & 123 & 51744 & 90000 & 71 & +52 \\
\hline Obstétrique-gynécologie & 399 & 15951 & 17500 & 364 & +35 \\
\hline Ophtalmologie & 214 & 29741 & 28000 & 227 & -13 \\
\hline 0to-rhino-laryngologie & 172 & 37003 & 50000 & 127 & +45 \\
\hline Pêdiatrie & 378 & 16837 & 20000 & 318 & +60 \\
\hline Physiatrie & 53 & 120085 & 100000 & 64 & -11 \\
\hline Psychiatrie & 650 & 9792 & 11000 & 579 & +71 \\
\hline Radiologie diagnostique & 389 & 16361 & 15300 & 416 & -27 \\
\hline Radiolonie therapeutique & 26 & 244788 & 154000 & 41 & -15 \\
\hline Urologie & 125 & 50916 & 50000 & 127 & -2 \\
\hline Santế communautaire & 91 & 69940 & 180000 & 35 & +56 \\
\hline TOTAL - SPEC IALISTES & $5668(2)$ & $1123(3)$ & $1232(4)$ & 5164 & +504 \\
\hline TOTAL - OMNIPRATICIENS & 5505 & 1156 & $1440(4)$ & 4419 & +1186 \\
\hline
\end{tabular}

Sources: MINISTERE DES AFFAIRES SOCIALES, L'offre et la demande de médecins spécialistes par spécialité au Québec. Etat des surplus et penuries 1980-1990. Jacques La haye, mai 1981, p. 37.

Tableau 1 (pour les omnipraticiens).

(1) Médecine interne regroupée comprend les spécialitês suivantes: Allerạie, cardiologie, endocrinologie, hếmatologie, gastro-entérologie, néphrologie, pneumologie et rhuma tologie.

(2) Il s'agit des médecins specialistes inscrits a la Corporation professionnelle des médecins du Quebec. Le total est différent de celui du tableau 1 quit correspond aux médecins spécialistes ayant reçu des revenus de la R.A.M.Q.

(3) Ratio population/medecin calcule avec le nombre de medecins inscrits a la Corporation professionnelle des medecins du Québec. Le ratio des spécialistes $(1: 1147)$ du tableau 1 est calcule avec le nombre de médecins inscrits a la Régie de l'assurance maladie du Québec (R.A.M.Q.).

(4) Population par médecin en 1981 telle que recommandée par le Comité national de la main-d'oeuvre médical. 
Même si l'on note une amélioration sensible du ratio population/médecin entre 1972 et 1980 (de 2033 à l 156), il n'y a pas eu d'amélioration relative de la distribution régionale des médecins omnipraticiens, malgré l'arrivée de 2500 nouveaux généralistes sur le marché du travail durant cette période (tableau 1). En 1980, il y avait près de deux fois plus de médecins omnipraticiens, au prorata de la population, sur l'Ile de-Montréal et à Laval (région 06A) qu'au Nord Ouest (région 08), soit une situation analogue à celle de 1972 .

L'addition de 1477 (36\%) médecins spécialistes de 1972 à 1980 n'aura concouru qu'à augmenter les disparités régionales: il y avait quatre fois moins de spécialistes au Nord Ouest (région 08) que dans les Cantons de-1'Est (région 05) en 1972, alors qu'en 1980, on en comptait cinq fois moins. Le problème de la répartition géographique des médecins spécialistes s'est donc aggravé au cours de la dernière décennie (tableau 1 ).

\subsection{Disparités_intrarágionales}

Le problème de la répartition géographique des médecins ne s'arrête cependant pas aux disparités interrégionales; c'est au niveau intrarégional qu'il prend toute sa signification. Les écarts intrarégionaux sont énormes, même dans certaines régions en surplus ou en équilibre par rapport à la norme. Par exemple en 1980, dans la région 6C (Montérégie), le ratio population/médecin omnipraticien est de 1 pour 1434 habitants par rapport à la norme fédérale de 1 pour 1440 .

Cependant une étude détailée dans chacun des 25 districts de C.L.S.C. qui composent cette région montre un portrait fort différent: parmi ces 25 districts, 12 connaissent une pénurie de 85 médecins omnipraticiens, alors que 11 présentent un surplus de 84 généralistes. Dans le cas des effectifs médicaux, une région en équilibre n'indique donc pas pour autant qu'il y ait équilibre intrarégional. Il faudra garder à l'esprit cette nuance lorsqu'il s'agira d'examiner la mise en place des mécanismes pour améliorer la répartition géographique des médecins.

1.3 Les - pénuries

On pourrait résumer brièvement cette analyse. D'une part, le nombre de médecins au Québec est généralement très satisfaisant si on compare le ratio population/médecin à celui des autres provinces canadiennes ou à celui des autres pays industrialisés (tableau 3). Par rapport à des normes fédérales établies en 1975 pour l'année 1981 (tableau 2), nous avons vu précédemment qu'il y avait un surplus global d'environ 1000 omnipraticiens et 500 spécialistes. Ce surplus théorique n'existe que dans trois régions du Québec, soit celles de Montréal Métropolitain, de Québec et des Cantons de-1'Est; toutes les autres régions connaissent des pénuries plus ou moins importantes malgré une croissance nette de 3900 médecins au Québec entre 1972 et 
TABLEAU 3

Rapport population/médecin (1) pour

le Québec et certains pays, 1976

\section{Pays}

Québec (2)

Canada

U.S.A.

Emirats Arabes Unis

U.R.S.S.

Grèce

Belgique

République fédérale allemande

Danemard

République démocratique allemande

Suisse

Norvège

Suède (1975)

Pays Bas

France (1975)

Japon
Rapport

556

580

600

230

300

470

500

500

510

520

520

560

580

600

650

850

Source: 0.M.S., Annuaire de statistiques sanitaires mondiales: personnel de santé et établissement hospitalier, Vol. III, 1978, Genève 1979, 85 p.

(1) Incluant les omnipraticiens, les spécialistes, les internes et les résidents.

(2) SANTE ET BIEN ETRE SOCIAL, CANADA, Répertoire de la main d'oeuvre sanitaire du Canada, 1978, juin 1979, p. 181 .

1980 (tableau 1). Ces constatations nous ont permis de cerner le problème dans les régions éloignées, non pas en terme de pénurie globale de ressources humaines, mais uniquement en terme de mauvaise répartition géographique.

Face à cette situation, le Ministère a cherché à mettre en place une politique de répartition qui tiendrait compte des réalités spécifiques de chacune des régions du Québec tout en assurant le plus possible à toute la population un égal accès aux soins. En effet, le Québec possède toutes les ressources humaines nécessaires pour mettre en oeuvre ce principe d'accessibilité: le défi consistait à arriver à 
modifier la répartition régionale des médecins. Il s'avérait dès lors nécessaire d'identifier et d'analyser les principales raisons qui ont engendré, au fil des ans, cette répartition inadéquate.

2. Les causes de la mauvaise répartition géographique des médecins

Les médecins ne sont pas des employés de l'Etat au même titre que les enseignants par exemple. Ce sont des entrepreneurs libres qui s'installent là où ils veulent, selon leur goat, et qui travaillent habituellement à leur compte, en rendant des services assurés par la Régie de l'assurance-maladie du Québec. Ce sont eux qui déterminent généralement leur niveau d'activité en fonction de leur clientèle et du niveau de revenu souhaité.

Ce libre choix du lieu d'exercice est la cause principale de la mauvaise répartition géographique des médecins. Dans le passé, aucune politique de main d'oeuvre ne tenait compte de la répartition optimale des effectifs de ce secteur d'activité à cause de ce libre choix. Mais cela ne dit pas pourquoi le libre choix entraine inévitablement une mauvaise répartition. Il faut savoir en plus:

- que le choix du lieu d'exercice d'un médecin n'est pas si libre qu'on pourrait le croire car il est fortement conditionné par des facteurs de civilisation, tel l'attrait des grands centres urbains;

- qu'une conjoncture de facteurs propre au domaine de la santé fait en sorte qu,il y a peu de contrepoids à ce conditionnement (rémunération, isolement scientifique, pratique plus exigeante, etc.).

Par ailleurs, d'autres facteurs ont accentué, au cours des ans, les causes de la répartition géographique inégalitaire des médecins. Parmi ces facteurs, on peut citer:

- la liberté totale d'accès au marché;

- une politique longtemps conciliante à l'égard des immigrants professionnels qui ont une très forte propension à exercer dans les grands centres urbains, là où les surplus sont énormes;

Si les surplus généraux qui s'accroissent sans cesse se répartissaient équitablement, il y aurait moins lieu de s'alarmer. Mais le système actuel fait en sorte que ceux ci se traduisent par des surplus territoriaux encore plus considérables, à cause de la gratuité des services, du libre choix du lieu d'exercice et de l'influence du médecin dispensateur qui crée en partie la demande en raison de la

(3) Ce chapitre est tiré de: MINISTERE DES AFFAIRES SOCIALES, Rapport du groupe de travail sur la répartition géographique des professionnels de la santé, aoat 1981, $147 \mathrm{p}$. 
rémunération à l'acte. On peut se demander si l'accroissement éventuel des concentrations de dispensateurs n'entrainera pas, tant au point de vue de la santé que de l'équilibre budgétaire, la présence accrue de certains phénomènes indésirables tels:

- la surdispensation;

- la médicalisation de la santé;

- la médecine "industrielle" (à la chaine);

- la recherche de nouveaux débouchés qui ne sont pas nécessairement prioritaires;

- la surutilisation des ressources hospitalières.

Comme les surplus d'effectifs favorisent la surdispensation de soins et d'autres phénomènes indésirables dans les grands centres urbains, et comme les pénuries réduisent l'accessibilité aux soins dans les régions périphériques et éloignées, le problème de la répartition géographique des médecins concerne donc la plus grande partie du territoire québécois. Voilà pourquoi la politique de répartition géographique vise à influencer, par certaines mesures, le choix du lieu d'exercice des médecins dans toutes les régions du Québec. Les unes favorisent l'installation de médecins dans les régions en pénurie, les autres découragent leur installation dans les régions en surplus.

\section{Les solutions envisagées}

Pour assurer une meilleure répartition géographique des médecins, on a étudié différents scénarios, qui vont des mesures incitatives aux mesures coercitives. Par exemple, différentes mesures incitatives ont été envisagées pour améliorer la situation des médecins dans les régions de pénurie dont:

- le remboursement de frais de perfectionnement;

- les primes d'éloignement;

- les primes d'installation;

- les tarifs différentiels selon les régions;

- le congé sabbatique.

D'autres mesures, plus coercitives, ont été considérées dont le contingentement régional de la participation au régime d'assurance maladie.

Enfin certaines mesures complémentaires ont également été étudiées, dont la décentralisation de la formation par des stages en régions éloignées et l'admission prioritaire pour les candidats provenant de ces régions et rencontrant les critères d'excellence. 


\section{La politique de répartition géographique des médecins}

\subsection{Principes}

Le Gouvernement a décidé de mettre en place une politique de répartition géographique des médecins omnipraticiens et spécialistes qui tienne compte des deux problèmes identifiés:

- surplus relatifs d'effectifs dans les grands centres urbains;

- pénuries relatives d'effectifs dans les régions éloignées.

Cette politique vise essentiellement à réduire l'attrait des régions en surplus et à accroitre celui des régions en pénurie en modifiant les comportements se rattachant au plan de carriere et à l'installation des médecins dans un territoire d'exercice. Le principe de la rémunération différentielle a été envisagé pour compenser, par des écarts de tarifs, les différences d'attrait des territoires d'exercice.

\subsection{Modalités}

Le mécanisme de la rémunération différentielle prévoit des tarifs majorés, réguliers ou réduits selon le lieu de dispensation des services médicaux et selon qu'il s'agit de médecins débutants ou déjà inscrits dans le régime d'assurance-maladie du Québec. De plus, des mesures incitatives complémentaires se sont ajoutées à la rémunération différentielle pour favoriser l'installation de nouveaux médecins dans les régions en pénurie et inciter ceux déjà installés à y demeurer.

Comme la Loi sur l'assurance maladie ne permettait pas au ministre des Affaires sociales de conclure des ententes prévoyant une rémunération différente selon le lieu d'exercice ou le nombre d'années d'expérience des médecins, il fallait donc, pour mettre en place cette politique, modifier cette loi.

En décembre 1981, la loi 27, prévoyant diverses dispositions législatives dans le domaine de la santé et des services sociaux, venait modifier la Loi sur l'assurance-maladie en ajoutant à l'article 19 de cette loi, les paragraphes suivants:

"Une entente peut prévoir une rémunération différente pour la fourniture de services médicaux dans un territoire où le ministre estime que les effectifs de professionnels de la santé sont insuffisants.

Elle peut également prévoir une rémunération différente pour les médecins durant les premières années d'exercice de leur profession ou de leur 
spécialité dans le cadre du régime, selon le territoire où ils exercent ou le genre d'activité qu'ils exercent." (4)

Pour mettre en place ce mécanisme prévu dans la loi, on a identifié les territoires insuffisamment pourvus de médecins et ceux en surplus par une évaluation du nombre de médecins requis selon la moyenne provinciale (5) par rapport aux effectifs observés en 1980.

Les résultats de ces travaux ont été soumis pour consultation aux organismes concernés par le problème de la répartition géographique des effectifs médicaux (Ministère des Affaires sociales, 1982).

\subsection{Regroupement_des_régions}

L'analyse statistique a démontré que les trois régions sociomsanitaires (6) suivantes connaissaient des surplus relatifs d'effectifs autant pour les médecins omnipraticiens que pour les médecins spécialistes: Montréal Métropolitain, Québec et Cantons de d'Est.

Par ailleurs, les quatre régions socio sanitaires (7) suivantes étaient dans une situation de pénurie relative d'effectifs médicaux: BasuSt-Laurent-Gaspésie, Nord Ouest, Côte Nord et Nouveau Québec.

Les cinq autres régions intermédiaires étaient dans une situation d'équilibre relatif: Saguenay Lac St-Jean, Trois Rivières, Laurentidesulanaudière, Montérégie et Outaouais.

(4) L.R.Q., c. A 29, art. 19.

(5) Ratio population/médecin en 1980. Pour les spécialistes des régions universitaires, les effectifs sont de $20 \%$ plus élevés que ceux requis selon la moyenne provinciale et de $20 \%$ moins élevés dans les autres régions. Ce pourcentage d'augmentation tient compte des facteurs pouvant justifier le besoin plus grand de spécialistes dans les régions universitaires pour l'enseignement et la recherche. Dans les autres régions, la faible densité de population et l'absence de recherches requièrent un moins grand nombre de spécialistes.

(6) Ces trois régions sont celles où on retrouve les quatre facultés de médecine. Dans la suite, nous utiliserons donc le terme "régions universitaires" pour les désigner.

(7) Par la suite, nous utiliserons le terme "régions excentriques" pour les désigner. 
L'analyse statistique intrarégionale a montré qu'un découpage par région socio sanitaire ne correspondait pas à la réalité. par exemple, la région de Québec est dans une situation de surplus relatif d'effectifs médicaux. Cependant, certains territoires de cette région (Rivière du Loup, St-Georges de Beauce) se rapprochent davantage de la situation observée dans les régions intermédiaires car ils connaissent de légères pénuries de médecins.

Il a donc fallu procéder à un découpage territorial qui corresponde mieux à la situation observée (tableau 4). Ainsi, en regroupant les régions et les parties de région qui présentaient des caractéristiques homogènes quant au ratio population/médecin et à l'attrait qu'elles exercent sur les médecins, on a identifié cinq groupes de régions (8):

ATTRAIT POUR

LES MEDECINS

1. Régions universitaires:

Montréal-Métropolitain (06A), Québec et Cantons de l'Est universitaires (parties de 03 et 05). Ce sont des régions en surplus relatifs d'effectifs dont l'attrait est très grand sur les médecins.

2. Périphérie des régions universitaires:

Québec (partie de 03), Cantons de l'Est (partie de 05), Laurentides Lanaudière (06B) et Montérégie (06C). Ces régions sont relativement en équilibre; elles attirent moins les médecins que les régions universitaires, mais plus que les autres régions.

3. Régions des agglomérations non universitaires de 100000 habitants et plus (9):

Saguenay Lac-St Jean (02), Trois Rivières (04) et Outaouais (07). Ce sont des régions relativement en équilibre, moins attrayantes que les deux groupes précédents à cause de l'éloignement des centres universitaires.

(8) Voir la carte en annexe.

(5) Régions intermédiaires. 
TABLEAU 4: NOMBRE DE MEDECINS OMNIPRATICIENS ACTIFS ${ }^{(1)}$ ET NOMBRE CALCULE SELON LA MOYENNE PROVINCIALE, PAR REGION ET REGROUPEMENT DE REGIONS, QUEBEC 1980

\begin{tabular}{|c|c|c|c|}
\hline REGROUPEMENT DE REGIONS & $\begin{array}{l}\text { NOMBRE DE } \\
\text { MEDECINS } \\
\text { ACTIFS (1) } \\
\text { A }\end{array}$ & $\begin{array}{l}\text { NOMBRE REQUIS } \\
\text { SELON LE MOY- } \\
\text { ENNE PROVIN- } \\
\text { CIALE } 1980 \\
1: 1575 \text { (2) }\end{array}$ & $\begin{array}{l}\text { ECART } \\
\text { A-B }\end{array}$ \\
\hline \multicolumn{4}{|l|}{ Régions universitaires } \\
\hline 03- Québec universitaire & 513 & 429 & +84 \\
\hline 05- Cantons-de-1'Est universitaire & 107 & 98 & +9 \\
\hline $\begin{array}{l}\text { 06A- Montréal métropolitain et } \\
\text { Laval }\end{array}$ & 1353 & 1344 & +9 \\
\hline $\begin{array}{l}\text { Total régions universitaires } \\
\text { Périphérie des régions universi- } \\
\text { taires }\end{array}$ & 1973 & 1871 & +102 \\
\hline 03- Québec périphérique & 203 & 190 & +13 \\
\hline $05-$ Cantons-de-1'Est périphérique & 45 & 48 & -3 \\
\hline 06B- Laurentides-Lanaudière & 301 & 288 & +13 \\
\hline 06C- Montérégie & 582 & 617 & -35 \\
\hline $\begin{array}{l}\text { Total périphérie des régions } \\
\text { universitaires } \\
\text { Régions intermédiaires }\end{array}$ & 1131 & 1143 & -12 \\
\hline 02- Saguenay-Lac St-Jean & 168 & 179 & -11 \\
\hline 04- Trois-Rivières & 233 & 271 & -38 \\
\hline 07- Outaouais & 147 & 172 & -25 \\
\hline Total des régions intermédiaires & 548 & 622 & -74 \\
\hline \multicolumn{4}{|l|}{ Régions excentriques } \\
\hline 01- Bas-St-Laurent-Gaspésie & 142 & 145 & -1 \\
\hline 08- Nord-Ouest & 74 & 96 & -22 \\
\hline 09- Côte-Nord & 58 & 76 & -18 \\
\hline Total régions excentriques & 274 & 317 & -43 \\
\hline $\begin{array}{l}\text { Régions inconnues et Nouveau- } \\
\text { Québec }\end{array}$ & 29 & - & +29 \\
\hline TOTAL & 3955 & 3953 & $-2^{(3)}$ \\
\hline
\end{tabular}

SOURCES; R.A.M.Q., Fichiers des professionnels de la santé et de la rémunération, 1980.

(1) Sont considérés comme actifs les médecins ayant reçu de la R.A.M.Q. des revenus de $25000 \$$ ou plus en 1980 .

(2) Le ratio (1:1575) est calculé avec les médecins omnipraticiens actifs (+25 $000 \$)$. Le ratio (1:1156) des tableaux 1 et 2 a été calculé avec 1 'ensemble des médecins omnipraticiens.

(3) La différence entre les colonnes A et B résulte des arrondis. 
ATTRAIT POUR

LES MEDECINS

4. Régions excentriques:

Bas-St-Laurent-Gaspésie (01), Nord Ouest (08) et Côte Nord (09). Ces trois régions ont une pénurie relative d'effectifs et elles attirent peu de médecins.

5. Secteurs isolés:

Nouveau Québec (10) et certaines localités isolées (Fermont, Gagnon, Iles derlam Madeleine, Chibougamau, etc.).

Pour chaque groupe de régions, les surplus et les pénuries sont donnés au tableau 5 .

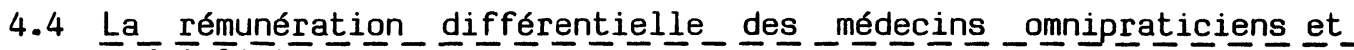
spécialiastes

La politique sur la rémunération différente est entrée en vigueur le 9 juin 1982 pour les médecins débutants et le ler juillet 1982 pour les médecins non débutants.

La rémunération différentielle prévoit une tarification réduite pour les médecins omnipraticiens et les médecins spécialistes de certaines spécialités (10), durant les trois premières années

(10) Cette tarification s'applique à 19 des 31 spécialités reconnues au Québec: la chirurgie générale, l'anesthésie, la radiologie diagnostique, la pédiatrie, la psychiatrie, l'obstétrique gynécologie, la médecine interne, l'anatomopathologie, la cardiologie, la chirurgie orthopédique, l'ophtalmologie, l'oto-rhino-laryngologie, l'urologie, l'hématologie, la neurologie, la dermatologie, la pneumologie, l'endocrinologie et la gastromentérologie. Elle vise à influencer le choix du lieu d'exercice des médecins de ces spécialités que l'on veut mieux répartir dans toutes les régions du Québec. L'installation en permanence de ces spécialistes dans les régions en pénurie réduira les besoins des médecins itinérants qui, quelques fois par année, fournissent des soins à la population de ces régions.

Les 12 autres spécialités ne sont pas touchées par la politique de la répartition géographique. Ce sont des surspécialités et il est préférable qu'elles se développent dans les grands centres urbains là où le volume de la clientèle est suffisant et où l'environnement est plus propice à la recherche et au développement scientifique. 
OMNIPRATICIENS

\begin{tabular}{|c|c|c|c|c|}
\hline REGROUPEMENT DE REGIONS & $\begin{array}{l}\text { NOMBRE DE } \\
\text { MEDECINS } \\
\text { ACTIFS(1) } \\
\text { (a) }\end{array}$ & $\begin{array}{c}\text { NOMBRE DE } \\
\text { MEDECINS REQUIS } \\
\text { SELON LA MOYENNE } \\
\text { PROVINCIALE } \\
1: 1575(2) \\
\text { (b) }\end{array}$ & $\begin{array}{l}\text { PENURIE }(-) \\
\text { OU } \\
\text { SURPLUS }(+) \\
\quad(c=a-b)\end{array}$ & $\begin{array}{c}\text { POURCENTAGE } \\
\text { DES } \\
\text { PENURIES (-) } \\
\text { OU } \\
\text { SURPLUS (+) } \\
\left(d=\frac{c}{b} \times 100\right)\end{array}$ \\
\hline $\begin{array}{l}\text { Régions universitaires (par- } \\
\text { ties de } 03 \text { et } 05 \text {, et } 05 \cdot A \text { au } \\
\text { complet) }\end{array}$ & 1973 & 1871 & +102 & $+5,5$ \\
\hline $\begin{array}{l}\text { Péripherie des regions uni- } \\
\text { versitaires (parties de } 03 \\
\text { et } 05,06 \text { B et } 06-C \text { au } \\
\text { complet }\end{array}$ & 1131 & 1143 & -12 & $-1,0$ \\
\hline $\begin{array}{l}\text { Regions intermediaires (02, } \\
04 \text { et } 07)\end{array}$ & 548 & 622 & -74 & $-11,9$ \\
\hline $\begin{array}{l}\text { Régions excentriques }(01,08 \\
\text { et 09) }\end{array}$ & 274 & 317 & & $-13,6$ \\
\hline $\begin{array}{l}\text { Régions inconnues et Nouveau- } \\
\text { Québec }\end{array}$ & 29 & - & +29 & - \\
\hline TOTAL & 3955 & 3953 & $-2^{(3)}$ & - \\
\hline
\end{tabular}

ENSEMBLE DES 19 SPECIALITES DESIGNEES ${ }^{(4)}$

\begin{tabular}{|c|c|c|c|c|}
\hline REGROUPEMENT DE REGIONS & $\begin{array}{l}\text { NOMBRE DE } \\
\text { MEDECINS } \\
\text { ACTIFS ( } 1) \\
(a)\end{array}$ & $\begin{array}{l}\text { NOMBRE DE } \\
\text { MEDECINS REQUIS } \\
\text { SELON LA MOYENNE } \\
\text { PROVINCIALE } \\
\text { CORRIGEE } \\
\text { (b) }\end{array}$ & $\begin{array}{c}\text { PENURIE }(-) \\
\text { OU } \\
\text { SURPLUS }(+) \\
(c=a-b)\end{array}$ & $\begin{array}{c}\text { POURCENTAGE } \\
\text { DES } \\
\text { PENURIES (-) } \\
\text { OU } \\
\text { SURPLUS (+) } \\
\left(d=\frac{c}{b} \times 100\right)\end{array}$ \\
\hline $\begin{array}{l}\text { Régions universitaires (par- } \\
\text { ties de } 03 \text { et } 05 \text {, et } 06 \mathrm{~A} \text { au } \\
\text { complet }\end{array}$ & 3181 & 2517 & +664 & $+26,4$ \\
\hline $\begin{array}{l}\text { Périphérie des régions uni- } \\
\text { versitaires (parties de } 03 \text { et } \\
05,06 \mathrm{~B} \text { et } 06 \mathrm{C} \text { au complet) }\end{array}$ & 576 & 972 & -396 & $-40,7$ \\
\hline $\begin{array}{l}\text { Régions in termédiaires } \\
(02,04 \text { et } 07)\end{array}$ & 391 & 550 & -159 & $-28,9$ \\
\hline $\begin{array}{l}\text { Régions excentriques } \\
(01,08 \text { et } 09)\end{array}$ & 128 & 282 & -154 & $-54,6$ \\
\hline $\begin{array}{l}\text { Régions inconnues et Nouveau- } \\
\text { Québec }\end{array}$ & 38 & - & +38 & - \\
\hline TOTAL & $4314^{(4)}$ & 4321 & $-7^{(3)}$ & - \\
\hline
\end{tabular}

Source: Evaluation des territoires en surplus et en pénurie dans l'état actuel de la répartition géographique des effectifs médicaux. Document de consultation,

D.G.R.T., ministère des Affaires sociales, février 1982, pages 16 et 17.

(1) Sont considérés comme actifs les médecins ayant reçu de la R.A.M.Q. des revenus de $25000 \$$ ou plus en 1980.

(2) Le ratio (1:1575) est calcule avec les médecins omnipraticiens actifs (+25 $000 \$)$. Le ratio $(1: 1156)$ des tableaux 1 et 2 a eté calcule avec 1 'ensemble des médecins omnipraticiens.

(3) La différence entre les colonnes (a) et (b) resulte des arrondis.

(4) Pour les 19 spécialités désignés sur les 31 spécialités reconnues par la Corporation professionnelle des médecins du Québec. 
d'exercice, s'ils s'installent dans les territoires désignés comme étant en surplus. Des tarifs majorés sont prévus pour les médecins déjà en pratique dans les territoires insuffisamment pourvus de professionnels et pour les nouveaux médecins qui s'y installent (11).

Le niveau de rémunération pour les spécialistes (débutants et non débutants) qui exercent dans les régions éloignées s'établit à 120\% de la rémunération de base. Pour les médecins omnipraticiens non débutants, la rémunération est majorée à 115\% alors que pour les omnipraticiens débutants, ce niveau est atteint à la deuxième année d'exercice. Dans les secteurs isolés, le tarif majoré s'applique dès la première année (tableau 6 et carte en annexe).

Les médecins débutants (omnipraticiens et spécialistes) qui rendent des services dans les territoires considérés en surplus reçoivent $70 \%$ de la rémunération de base durant les trois premières années d'exercice.

Deux exceptions importantes sont prévues dans l'application de cette politique de répartition géographique:

1. Dans les régions périphériques de Montréal (Laurentides Lanaudière et Montérégie), les nouveaux médecins omnipraticiens et spécialistes qui exercent en établissement reçoivent la rémunération de base (100\%) alors que ceux qui travaillent hors établissement reçoivent 70\% de la rémunération de base pendant trois ans (voir le tableau 3 et la carte en annexe). Cette exception tient compte du problème particulier de ces deux régions périphériques de Montréal à savoir un nombre relativement satisfaisant de médecins en cabinet privé, mais des pénuries importantes d'effectifs médicaux en établissement.

2. Durant les premières années d'exercice, les médecins qui s'installent dans les territoires en surplus reçoivent la rémunération de base lorsqu'ils sont nommés, comme professeurs, par les facultés de médecine. Cette exception a été accordée afin que les universités puissent recruter de jeunes professeurs et éviter que les médecins désirant poursuivre une carrière de recherche et d'enseignement quittent le Québec.

Des mesures incitatives complémentaires s'ajoutent à la rémunération différentielle pour accroitre la capacité d'attirer et de retenir les médecins des régions éloignées et des secteurs isolés. Ces mesures sont:

- les frais de perfectionnement pour les médecins oeuvrant en régions insuffisamment pourvues;

(11) Les sommes récupérées dans les territoires désignés en surplus serviront à compenser les tarifs majorés pour les régions en pénurie. 
TABLEAU 6: NIVEAU DE REINNERATION PAR REGROUPEMENT DE REGIONS

\section{OMNIPRAIICIENS}

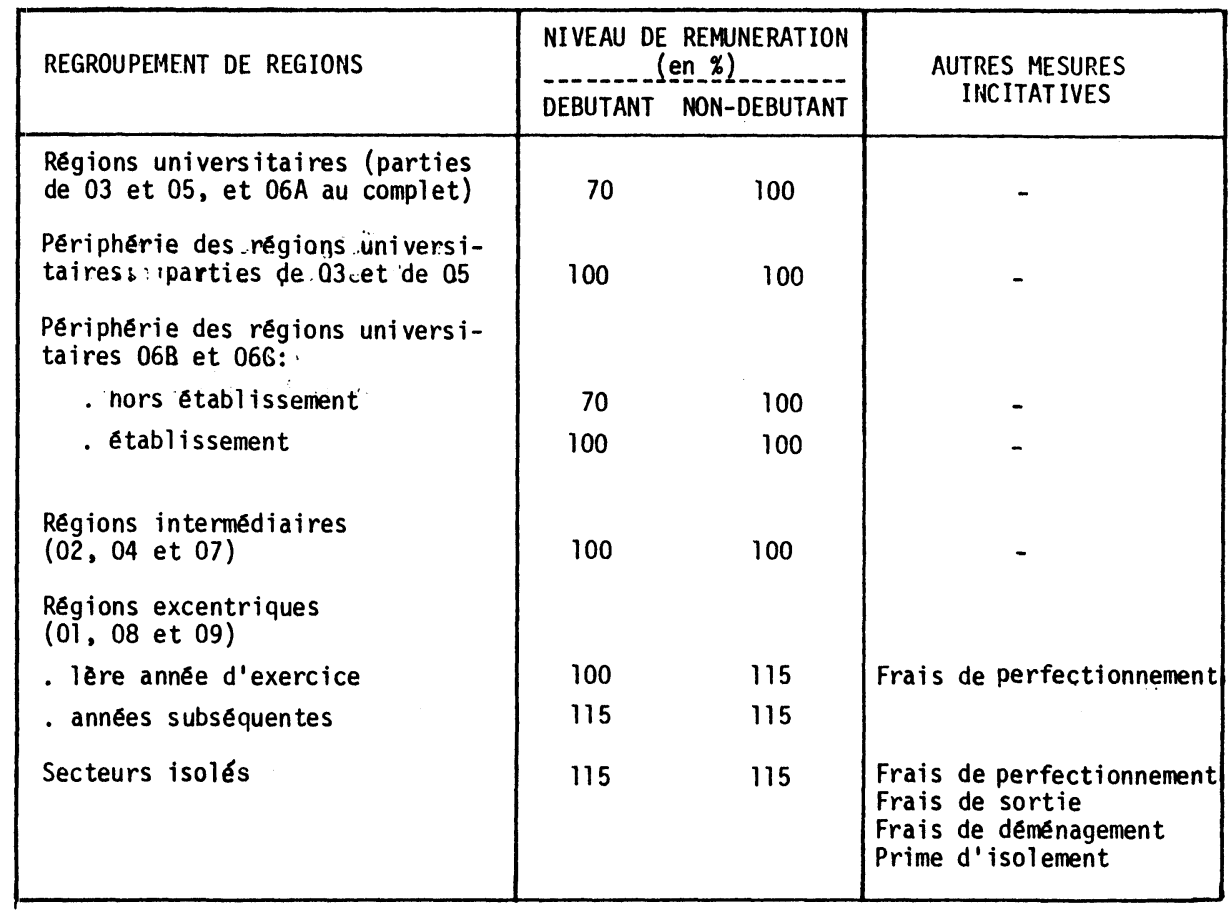

SPECIALISTES (19 SPECIALITES DESIGNEES)

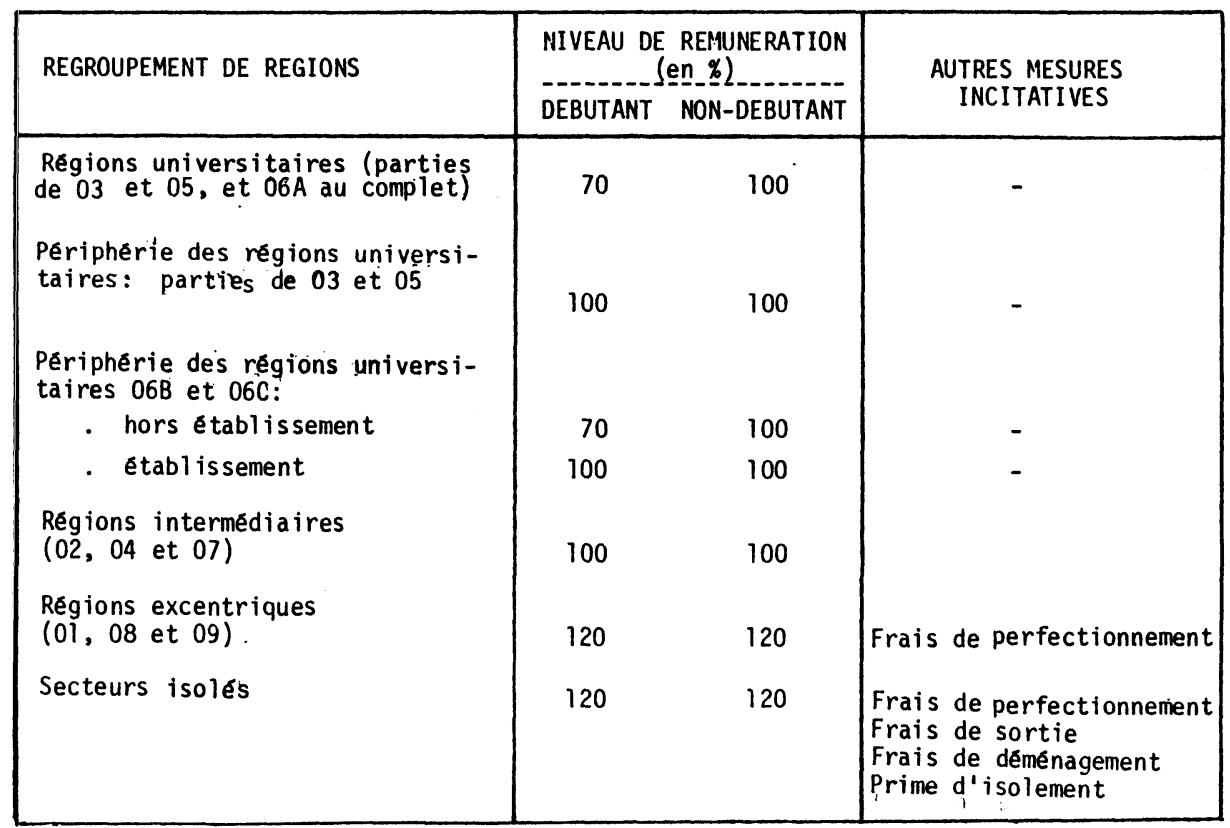


et plus spécifiquement pour les médecins choisissant de pratiquer dans les secteurs isolés:

- le remboursement des frais de déménagement;

- le remboursement des frais de sortie (transport vers Montréal ou Québec, trois fois par année);

- la prime d'éloignement ou d'isolement.

De plus, on a amélioré le programme de bourses pour les étudiants en médecine qui s'engagent à exercer leur profession dans des territoires désignés.

\section{Résultats préliminaires}

L'impact de cette politique sur la répartition géographique des médecins ne peut être évalué que partiellement pour le moment.

Les nouveaux médecins omnipraticiens ont obtenu leur permis d'exercice au moment où cette politique entrait en vigueur en juin 1982. La plupart d'entre eux avaient choisi leur lieu d'exercice depuis quelques mois. C'est pourquoi il $n^{\prime} y$ a pas eu de modifications importantes des choix habituels de ces lieux d'exercice en 1982 (tableau 7).

En 1983, les résultats sont plus concluants. L'installation dans les régions universitaires a baissé à 46,4\%. Par contre, les régions excentriques marquaient un léger recul par rapport à l'année précédente qui avait été une année exceptionnelle. Les régions intermédiaires ont doublé leur gain en 1982 et 1983 profitant de la diminution dans les régions universitaires.

Dans le cas des médecins spécialistes, il y a une baisse significative du pourcentage d'installation des médecins débutants dans les régions universitaires depuis 1982 au profit des régions intermédiaires. Les changements ne sont pas encore perceptibles dans les autres régions.

Dans l'ensemble, on observe à partir de 1982 une inversion de la tendance des années précédentes aussi bien dans le cas des omnipraticiens que des spécialistes. Ce changement amorcé depuis deux ans devrait se poursuivre et modifier substantiellement la distribution régionale des médecins afin d'atteindre les objectifs visés par la politique de répartition géographique des médecins. 
TABLEAU 7

Répartition des médecins omnipraticiens débutants par regroupement de régions, Québec 1978 à 1983

\begin{tabular}{|c|c|c|c|c|c|c|c|}
\hline \multirow{2}{*}{ REGROUPEMENT DE REGIONS } & & \multicolumn{6}{|c|}{ MEDECINS OMNIPRATICIENS (1) } \\
\hline & & 1978 & 1979 & 1980 & 1981 & 1982 & 1983 \\
\hline $\begin{array}{l}\text { Régions universitaires } \\
\text { (parties de } 03 \text { et } 05,06 \mathrm{~A} \text { au complet) }\end{array}$ & $\begin{array}{c}\% \\
(\mathrm{~N})\end{array}$ & $\begin{array}{r}51,3 \\
(155)\end{array}$ & $\begin{array}{r}53,4 \\
(147)\end{array}$ & $\begin{array}{r}58,9 \\
(133)\end{array}$ & $\begin{array}{r}58,8 \\
(137)\end{array}$ & $\begin{array}{r}55,4 \\
(123)\end{array}$ & $\begin{array}{r}46,4 \\
(90)\end{array}$ \\
\hline $\begin{array}{l}\text { Périphérie des régions universitaires } \\
\text { (parties de } 03 \text { et } 05,06 \mathrm{~B} \text { et } 06 \mathrm{C} \text { au complet) }\end{array}$ & $\begin{array}{c}\% \\
(\mathrm{~N})\end{array}$ & $\begin{array}{r}28,8 \\
(\quad 87)\end{array}$ & $\begin{array}{r}22,2 \\
(61)\end{array}$ & $\begin{array}{r}24,3 \\
(55)\end{array}$ & $\begin{array}{r}23,6 \\
(55)\end{array}$ & $\begin{array}{r}22,5 \\
(50)\end{array}$ & $\begin{array}{r}23,2 \\
(45)\end{array}$ \\
\hline $\begin{array}{l}\text { Régions intermédiaires } \\
(02,04 \text { et } 07)\end{array}$ & $\begin{array}{c}\% \\
(\mathrm{~N})\end{array}$ & $\left(\begin{array}{l}7,3 \\
(22)\end{array}\right.$ & $\begin{array}{r}16,7 \\
(46)\end{array}$ & $\begin{array}{r}10,2 \\
(23)\end{array}$ & $\begin{array}{r}12,0 \\
(\quad 28)\end{array}$ & $\begin{array}{r}9,5 \\
(21)\end{array}$ & $\begin{array}{r}20,6 \\
(40)\end{array}$ \\
\hline $\begin{array}{l}\text { Régions excentriques }(01,08 \text { et } 09) \\
\text { et secteurs isolés }\end{array}$ & $\begin{array}{c}\% \\
(\mathrm{~N})\end{array}$ & $\begin{array}{r}12,6 \\
(\quad 38)\end{array}$ & $\begin{array}{r}7,7 \\
(21)\end{array}$ & $\begin{array}{r}6,6 \\
(15)\end{array}$ & $\begin{array}{l}5,6 \\
(13)\end{array}$ & $\begin{array}{r}12,6 \\
(\quad 28)\end{array}$ & $\begin{array}{l}9,8 \\
(19)\end{array}$ \\
\hline TOTAL & $\begin{array}{c}\% \\
(\mathrm{~N})\end{array}$ & $\begin{array}{l}100,0 \\
(302)\end{array}$ & $\begin{array}{l}100,0 \\
(275)\end{array}$ & $\begin{array}{l}100,0 \\
(226)\end{array}$ & $\begin{array}{l}100,0 \\
(233)\end{array}$ & $\begin{array}{l}100,0 \\
(222)\end{array}$ & $\begin{array}{l}100,0 \\
(194)\end{array}$ \\
\hline
\end{tabular}

Source: MINISTERE DES AFFAIRES SOCIALES, Evaluation de 1'impact des mesures de répartition géographique, Direction de 1 'organisation des services de santé, Québec, avril $1984,22 \mathrm{p}$.

(1) A 1'exclusion des médecins omnipraticiens certifiés en médecine familiale et des boursiers de la R.A.M.Q.

TABLEAU 8

Répartition des médecins spécialistes débutant dans une spécialité désignée, par regroupement de régions, Québec 1978 à 1983

\begin{tabular}{|c|c|c|c|c|c|c|c|}
\hline \multirow{2}{*}{ REGROUP EMLNT DE REGIONS } & & \multicolumn{6}{|c|}{ MEDECINS SPECIALISTES } \\
\hline & & 1978 & 1979 & 1980 & 1981 & 1982 & 1983 \\
\hline $\begin{array}{l}\text { Régions universitaires } \\
\text { (parties de } 03 \text { et } 05,06 \mathrm{~A} \text { au complet) }\end{array}$ & $\begin{array}{l}\% \\
(\mathrm{~N})\end{array}$ & $\begin{array}{r}77,8 \\
(161)\end{array}$ & $\begin{array}{r}76,4 \\
(139)\end{array}$ & $\begin{array}{r}79,4 \\
(162)\end{array}$ & $\begin{array}{l}78,8 \\
(126)\end{array}$ & $\begin{array}{r}76,7 \\
(115)\end{array}$ & $\begin{array}{r}73,7 \\
(115)\end{array}$ \\
\hline $\begin{array}{l}\text { Périphérie des régions universitaires } \\
\text { (parties de } 03 \text { et } 05,06 \mathrm{~B} \text { et } 06 \mathrm{C} \text { au complet) }\end{array}$ & $(\mathrm{N})$ & $\begin{array}{r}6,3 \\
(13)\end{array}$ & $\begin{array}{r}13,7 \\
(25)\end{array}$ & $\begin{array}{c}10,8 \\
(22)\end{array}$ & $\begin{array}{r}13,8 \\
(22)\end{array}$ & $\begin{array}{r}10,0 \\
\left(\begin{array}{r}15 \\
)\end{array}\right.\end{array}$ & $\begin{array}{r}10,3 \\
(16)\end{array}$ \\
\hline $\begin{array}{l}\text { Régions intermédiaires } \\
(02,04,07)\end{array}$ & $\begin{array}{c}\% \\
(N)\end{array}$ & $\begin{array}{r}10,1 \\
(21)\end{array}$ & $\begin{array}{l}7,7 \\
(14)\end{array}$ & $\begin{array}{l}6,9 \\
(14)\end{array}$ & $\begin{array}{r}3,8 \\
\left(\begin{array}{r}6\end{array}\right)\end{array}$ & $\begin{array}{r}9,3 \\
(14)\end{array}$ & $\begin{array}{r}10,9 \\
(17)\end{array}$ \\
\hline $\begin{array}{l}\text { Régions excentriques }(01,08,09) \\
\text { et secteurs isolés }\end{array}$ & $\begin{array}{c}\% \\
(N)\end{array}$ & $\begin{array}{l}5,8 \\
(12)\end{array}$ & $\begin{array}{r}2,2 \\
(\quad 4)\end{array}$ & $\begin{array}{r}2,9 \\
(\quad 6)\end{array}$ & $\begin{array}{r}3,8 \\
(\quad 6)\end{array}$ & $\begin{array}{r}4,0 \\
(\quad 6)\end{array}$ & $\left(\begin{array}{r}5,1 \\
8\end{array}\right)$ \\
\hline TO'TAL & $\begin{array}{l}\% \\
\text { (N) }\end{array}$ & $\begin{array}{l}100,0 \\
(207)\end{array}$ & $\begin{array}{l}100,0 \\
(182)\end{array}$ & $\begin{array}{l}100,0 \\
(204)\end{array}$ & $\begin{array}{l}100,0 \\
(160)\end{array}$ & $\begin{array}{l}100,0 \\
(150)\end{array}$ & $\begin{array}{l}100,0 \\
(156)\end{array}$ \\
\hline
\end{tabular}

Source: MINISTERE DES AFFAIRES SOCIALES, Evaluation de 1'impact des mesures de répartition géographique, Di rection de $1^{\prime}$ organisation des services de santé, Québec, avril 1984, 22 p. 
RÉMUNÉRATION DIFFÉRENCIÉE DES MÉDECINS OMNIPRATICIENS ET SPÉCIALISTES

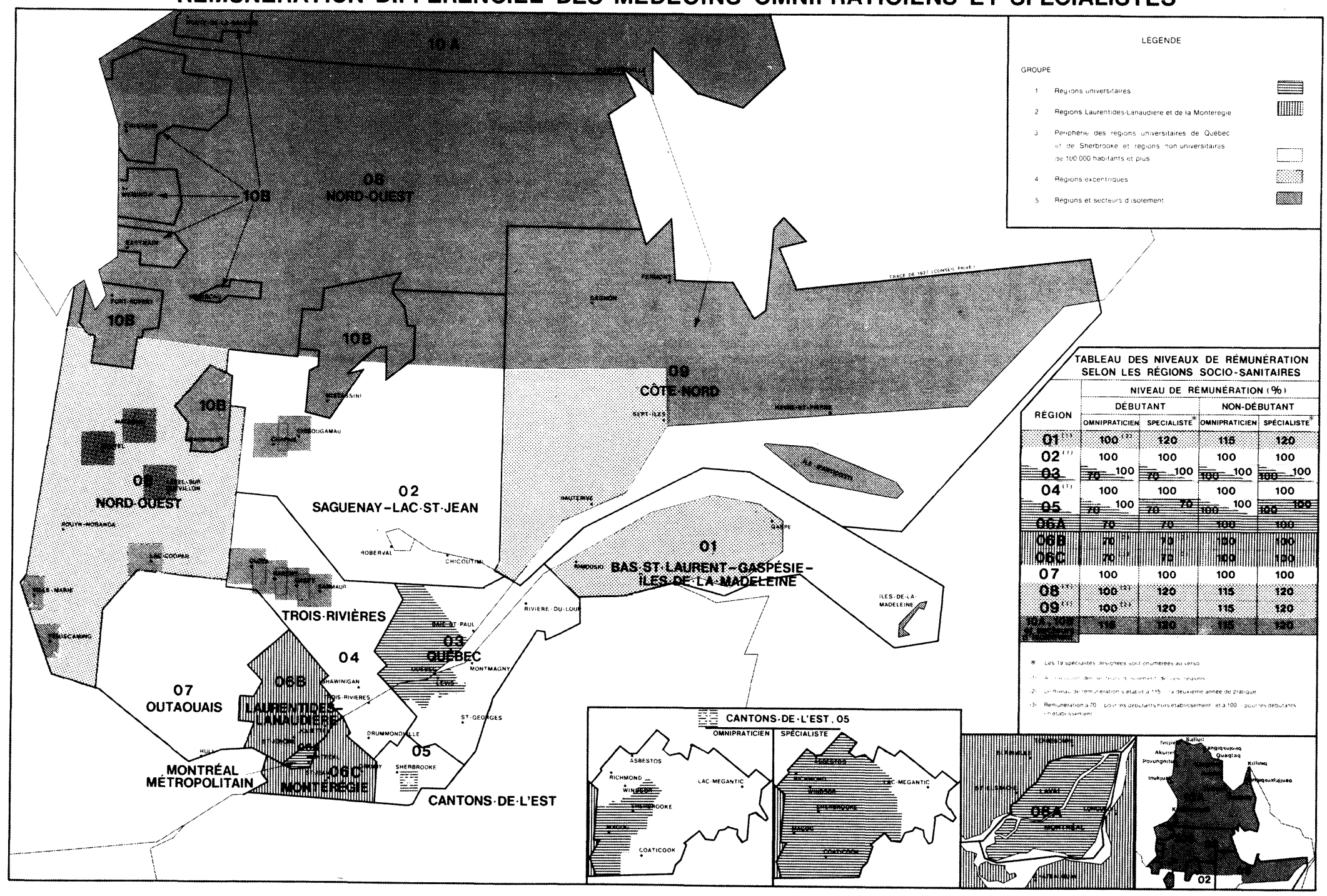


REFERENCES BIBLIOGRAPHIQUES

ASSOCIATION DES HOPITAUX DU QUEBEC, 1981. Problème des effectifs médicaux dans les régions périphériques: analyse et hypothèses de solutions, Montréal, $57 \mathrm{p}$.

BOUL.ARD, Richard, 1981. Les mécanismes de dépannage pour les médecins omnipraticiens et les spécialistes, document de travail, Québec, Service de planification et développement de la main d'oeuvre, Direction générale des relations de travail, Ministère des Affaires sociales, $18 \mathrm{p}$.

CANARIE, J.D. jr., 1980. "Maldistributed Health Care Services: Restructuring the Current Regulatory System", American Journal of Law and Medicine, 6, 3, 407-423.

COMITE NATIONAL DE LA MAIN D'OEUVRE MEDICALE, Comité des besoins, 1975. Rapport du Comité des besoins en main d'oeuvre médicale au Comité national de la main-d'oeuvre médicale, Ottawa, Ministère de la Santé nationale et du Bien Etre social, 3 tomes.

CONTANDRIOPOULOS, André-Pierre, PINEAULT, Raynald et FOURNIER, MarcAndré, 1982. Opinion des médecins québécois sur la pratique en région éloignée et sur le mode de rémunération, Faculté de médecine, Université de Montréal, $144 \mathrm{p}$.

COOPER, James K., HEALD, Karen, SAMUELS, Michael et COLEMAN, Sinclair, 1975. "Rural or urban Practice: Factors Influencing the Location Decision of Primary Care Physicians", Inquiry, 12, 18 25.

CORPORATION PROFESSIONNELLE DES MEDECINS DU QUEBEC, 1981. Les effectifs médicaux au Québec. Situation de 1978 à 1980 et projection pour 1984, Montréal, $118 \mathrm{p}$.

EISENBERG, B.S. et CANTWELL, J.R., 1976. "Policies to Influence the Spatial Distribution of Physicians: a Conceptual Review of Selected Programs and Empirical Evidence", Medical Care, 14, 6, 455 468.

GRADUATE MEDICAL EDUCATION NATONAL ADVISORY COMMITTEE, 1978. Physician Manpower Requirements, GMENAC Staff Paper 1, Washington, Department of health, Education and Welfare, $97 \mathrm{p}$.

HADLEY, J., 1979. "Alternative Methods of Evaluating Health Manpower Distribution", Medical Care, 17, 10, 1054-1060.

HALL, T.L. et MEJIA, A., 1979. La planification des professionnels de la santé: principes, méthodes, problèmes, Genève, Organisation mondiale de la santé.

"Health Manpower Shortage Areas, Criteria for Designation", Federal Register, 43, 6, Part 2, January 10, 1978, 1586 1596. 
KNOX, P.L., 1979. "Medical Deprivation, Area Deprivation and Public Policy", Social Science and Medicine (Medical Geography), 13D, 12 , $111-121$.

LA HAYE, Jacques, 1981. L'offre et la demande de médecins spécialistes au Québec. Etat des surplus et pénuries. 1980 1990, Québec, Service de planification et développement de la main d'oeuvre, Direction générale des relations de travail, Ministère des Affaires sociales, $98 \mathrm{p}$.

L.Q., 1981, c. 22.

MINISTERE DES AFFAIRES SOCIALES, 1982. Evaluation des territoires en surplus et en pénurie dans l'état actuel de la répartition géographique des effectifs médicaux, document de consultation, Québec, Direction générale des relations de travail, 74 p.

MINISTERE DE AFFAIRES SOCIALES, 1980. Politique du M.A.S. concernant la répartition géographique des professionnels de la santé, Québec, Direction générale des relations de travail, $70 \mathrm{p}$.

MINISTERE DES AFFAIRES SOCIALES, 1984. Evaluation de l'impact des mesures de répartition géographique, Québec, Direction de l'organisation des services de santé, $22 \mathrm{p}$.

MINISTERE DES AFFAIRES SOCIALES, 1981. Rapport du Comité interministériel sur le service communautaire, Montréal, Comité interministériel sur le service communautaire, $170 \mathrm{p}$.

MINISTERE DES AFFAIRES SOCIALES, 1981. Rapport du groupe de travail sur la répartition géographique des professionnels de la santé, Québec, Direction générale des relations de travail, $147 \mathrm{p.}+$ annexes.

MINISTERE DES AFFAIRES SOCIALES, 1980. La répartition territoriale des médecins omnipraticiens, des dentistes et des médecins spécialistes par municipalité - code postal, territoire de district de C.L.S.C., zone de D.S.C. et région socio sanitaire du Québec, document de référence no l, Québec, Service de l'évaluation, Direction générale des programmes de services sociaux, $295 \mathrm{p}$.

ONTARIO COUNCIL OF HEALTH, 1974. Physician Manpower, Toronto, Council of health, $62 \mathrm{p}$.

REGIE DE L'ASSURANCE MALADIE DU QUEBEC, Evolution du nombre de la répartition géographique et de la rémunération des professionnels de la santé, 1970-1972, Québec, $17 \mathrm{p}$.

SOUTHERN REGIONAL EDUCATION BOARD, 1978. Influencing the Distribution of Physicians: Manpower Policy Strategies, Atlanta, GA, Southern Regional Education Board, 8 p. 
TOURINIER, Pierre, 1977. "Evolution des disparités de la répartition géographique des médecins libéraux en France entre 1962 et 1975", Cahiers de sociologie et de démographie médicales, 17,4 , 131-151.

TURGEON, Jean, 1982. Situation actuelle et à venir des services itinérants et de dépannage dans la dispensation des services médicaux au Québec, Québec, Service de planification et développement de la main d'oeuvre, Direction générale des relations de travail, Ministère des Affaires sociales, $30 \mathrm{p.} \mathrm{+}$ annexes.

WENDLING, W.R., WERNER, J.L. et BUDDE, N.W., 1981. "Health Manpower Programs to Affect Physicians Location: a Regional Analysis", Journal of Health Politics, Policy and Law, 6, 1, 120 135. 
RESUME SUMMARY RESUMEN

BOULARD Richard et DUFOUR Desmond. - LA POLITIQUE DE REPARTITION GEOGRAPHIQUE DES EFFECTIFS MEDICAUX AU QUEBEC.

Un des objectifs du Ministère des Affaires sociales est d'assurer le plus possible à tous un égal accès aux soins de santé. Une évaluation quantitative de la répartition des médecins montre qu'il existe des disparités régionales importantes. Les médecins sont fortement concentrés dans les régions universitaires de Montréal, Québec et Sherbrooke, ce qui n'assure pas à la population de certaines régions périphériques l'accessibilité visée. Plusieurs solutions ont été envisagées à la suite de l'analyse des causes de ce déséquilibre régional des effectifs médicaux. Finalement, pour corriger cette situation, le Gouvernement a élaboré et mis en place une politique de répartition axée principalement sur une rémunération différente des médecins selon les lieux d'exercice. Quelques résultats préliminaires de cette politique sont présentés dans la dernière partie.

BOULARD Richard et DUFOUR Desmond. - THE POLICY OF GEOGRAPHICAL DISTRIBUTION OF QUEBEC'S MEDICAL STAFF.

One of the Ministry of Social Affairs' objectives is to insure, as much as possible, that everyone has equal access to medical care. A quantitative assessment of the distribution of doctors shows that there are significant regional disparities. Doctors are heavily concentrated in the university regions of Montréal, Québec City and Sherbrooke, which does not insure that the population of certain peripheral regions get the planned access to medical care. Several solutions have been considered, following the analysis of the regional unbalance of the medical staff. Finally, in order to correct this situation, the Government has developed and put in place a distribution policy mainly centered around a specific remuneration of doctors according to place of practice. Some preliminary results of this policy are presented in the last section.

BOULARD Richard et DUFOUR Desmond. - LA POLITICA DE DISTRIBUCION GEOGRAF ICA DEL PERSONAL MEDICO, EN QUEBEC.

Uno de los objetivos del ministerio de asuntos sociales es de asegurar un acceso libre e igualitario a los servicios de salud. La informacion disponible muestra que la distribucion espacial de los médicos contiene disparidades regionales importantes. Hay grandes concentrationes en las regiones universitarias (Montréal, Québec y Sherbrooke) mientras que los médicos se vuelven escasos en las regiones periféricas. Se han considerado varias soluciones posibles. Finalmente, el gobierno ha puesto en practica una politica de re distribucion sobre la base de una remuneracion diferencial, cuyos primeros resultados son presentados en la ultima parte. 